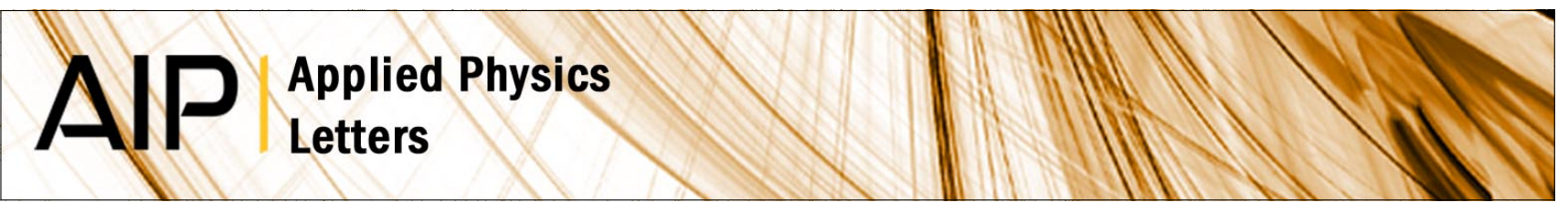

\title{
Single source coherent anti-Stokes Raman microspectroscopy using spectral focusing
}

Wolfgang Langbein, Israel Rocha-Mendoza, and Paola Borri

Citation: Appl. Phys. Lett. 95, 081109 (2009); doi: 10.1063/1.3216073

View online: http://dx.doi.org/10.1063/1.3216073

View Table of Contents: http://apl.aip.org/resource/1/APPLAB/v95/i8

Published by the American Institute of Physics.

\section{Related Articles}

Can stimulated Raman pumping cause large population transfers in isolated molecules?

J. Chem. Phys. 135, 184202 (2011)

Abnormal anti-Stokes Raman emission as single beam coherent anti-Stokes Raman scattering like process in

$\mathrm{LiNbO3}$ and CdS powder

J. Appl. Phys. 110, 053106 (2011)

Highly selective standoff detection and imaging of trace chemicals in a complex background using single-beam coherent anti-Stokes Raman scattering

Appl. Phys. Lett. 99, 101109 (2011)

Communication: Observation of homonuclear propensity in collisional relaxation of the 13C12CD2 (v2 = 1)

isotopologue of acetylene by stimulated Raman spectroscopy

J. Chem. Phys. 134, 231102 (2011)

Electronic-resonance-enhanced coherent anti-Stokes Raman scattering of nitric oxide: Saturation and Stark effects

J. Chem. Phys. 133, 084310 (2010)

\section{Additional information on Appl. Phys. Lett.}

Journal Homepage: http://apl.aip.org/

Journal Information: http://apl.aip.org/about/about_the_journal

Top downloads: http://apl.aip.org/features/most_downloaded

Information for Authors: http://apl.aip.org/authors

\section{ADVERTISEMENT}

\section{(@) ACCELERATE AMBER AND NAMD BY 5X. NVIDIA TRYIT ON A FREE, REMOTELYYHOSTED CLUSTER.}




\title{
Single source coherent anti-Stokes Raman microspectroscopy using spectral focusing
}

\author{
Wolfgang Langbein, ${ }^{1}$ Israel Rocha-Mendoza, ${ }^{1,2}$ and Paola Borri ${ }^{2, a)}$ \\ ${ }^{1}$ School of Physics and Astronomy, Cardiff University, The Parade, Cardiff CF24 3AA, United Kingdom \\ ${ }^{2}$ School of Biosciences, Cardiff University, Museum Avenue, Cardiff CF10 3AX, United Kingdom
}

(Received 28 June 2009; accepted 11 August 2009; published online 27 August 2009)

\begin{abstract}
We demonstrate coherent anti-Stokes Raman scattering (CARS) microspectroscopy using a single Ti:sapphire laser oscillator and simple passive optical elements. Spectral selection by dichroic mirrors and linear chirping by glass elements creates a vibrational excitation tuneable over a large spectral range $\left(\sim 800-2200 \mathrm{~cm}^{-1}\right)$ with adjustable spectral resolution $\left(\sim 10-100 \mathrm{~cm}^{-1}\right)$. We furthermore demonstrate the applicability of differential CARS, enhancing the chemical selectivity, with the proposed single-laser configuration. (C) 2009 American Institute of Physics.
\end{abstract}

[DOI: $10.1063 / 1.3216073]$

Coherent anti-Stokes Raman scattering (CARS) microscopy has been an active research field since its revival in 1999. ${ }^{1}$ This imaging technique joins the chemical sensitivity and label-free noninvasiveness offered by vibrational Raman scattering with the inherent three-dimensional sectioning capability of multiphoton microscopy (see Refs. 2-5 and references therein). However, the complexity and cost of the laser systems involved are major obstacles for the widespread application of CARS microscopy in medical imaging and material sciences. Raman resonances of interest have typically coherence times of the order of $1 \mathrm{ps}$, hence, their coherent excitation is optimized in spectral selectivity using picosecond pulses. Consequently the preferred laser systems for single-frequency CARS are picosecond sources, for example two electronically synchronized Ti:sapphire oscillators ${ }^{6}$ or an optical parametric oscillator pumped by a picosecond frequency-doubled $\mathrm{Nd}$ :vanadate laser. ${ }^{5}$ However, picosecond sources are less efficient for two-photon fluorescence (TPF) and second-harmonic generation (SHG) microscopy which otherwise would lend themselves to be implemented with CARS in a multimodal multiphoton microscope. It is possible to achieve high spectral selectivity in CARS using femtosecond pulses since the spectrum of the vibrational excitation is determined by the spectrum of their temporal interference, which can be tailored by controlling the pulse phase without energy loss. Having spectral selectivity in the excitation allows the detection of CARS by fast and efficient single-channel detectors as TPF and SHG microscopy, in contrast to spectral multiplexing techniques. ${ }^{7-9}$ An efficient way to reduce the spectral width of the pulse intensity was introduced to generate tuneable terahertz radiation, ${ }^{10}$ and termed "spectral focusing" for CARS. ${ }^{11}$ In this scheme, equal linear chirp is applied to Pump and Stokes pulses, creating a constant instantaneous frequency difference (IFD) between them, and we recently showed that glass dispersion can be used for that purpose. ${ }^{12}$ The resulting vibrational excitation is centered at the IFD and has a width given by the Fourier-limit of the temporal envelope of the pulses, which can be elongated by the applied chirp to many picoseconds. The IFD can be tuned by the Pump-Stokes time delay without tuning the laser sources. Thus, femtosecond pulses can

${ }^{a)}$ Electronic mail: borrip@cardiff.ac.uk. be used for tuneable single-frequency CARS, but still two synchronized laser pulses were needed in Refs. 11 and 12. A recent step forward ${ }^{13}$ to simplify the laser system uses spectral focusing with a $100 \mathrm{fs}$ Ti:sapphire oscillator as Pump and the continuum generated in a fiber by the same oscillator ${ }^{8}$ as Stokes. However, continuum generation in fibers is sensitive to laser fluctuations and alignment, and also suffers from aging. Furthermore, since the bandwidths of the oscillator and the continuum are different by an order of magnitude, the scheme is not efficient since only a small part of the continuum is creating CARS, while the rest is both wasting laser power and additionally exposing the sample.

In this paper, we demonstrate that a single broadband Ti:sapphire oscillator can be efficiently used to implement single and dual frequency CARS. The same source is suited for TPF and SHG, allowing for a cost-effective implementation of a multimodal multiphoton microscope.

A scheme of the optical setup is shown in Fig. 1. The laser source is a prism-compensated Ti:sapphire oscillator (KMLabs MTS) pumped by $4.5 \mathrm{~W}$ of a frequency doubled Nd:vanadate laser (Laser Quantum Finesse). Its bandwidth

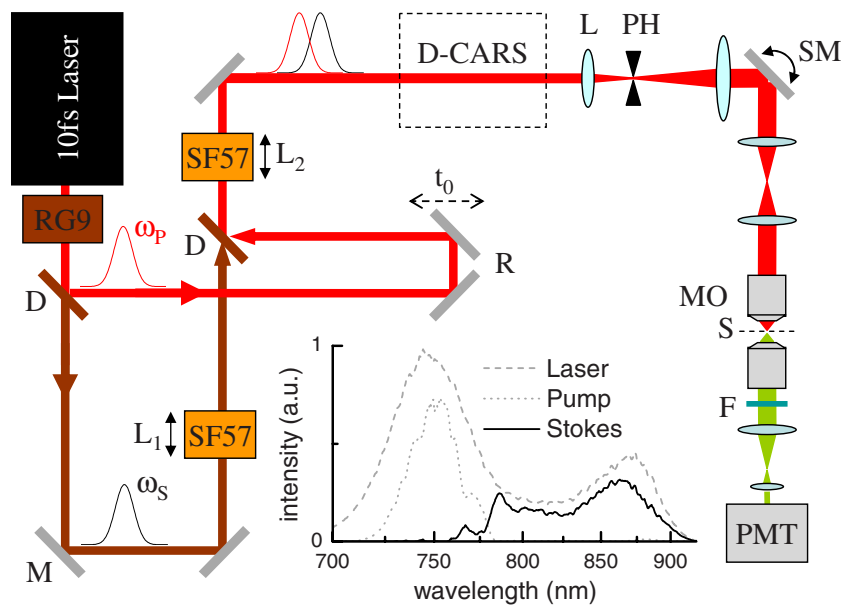

FIG. 1. (Color online) Sketch of the experimental setup. $\omega_{\mathrm{S}, \mathrm{P}}$ : Stokes and Pump beams, RG9: long pass filter, D: dichroic mirror, M: silver mirror, SF57: glass block, L: lens, R: retroreflector, PH: pinhole, SM: scanning mirror, MO: microscope objective, S: sample, F: bandpass filter, PMT: photomultiplier. D-CARS: differential CARS optics. Inset: typical spectra of laser, Pump, and Stokes. 
can be varied from $400 \mathrm{~cm}^{-1}$ (50 fs) to $2400 \mathrm{~cm}^{-1}$ ( $8 \mathrm{fs}$ ) using slit and prism positions, and it provides $300-500 \mathrm{~mW}$ of power at $95 \mathrm{MHz}$ repetition rate. To excite CARS of vibrational resonances in the characteristic region $\left(800-1800 \mathrm{~cm}^{-1}\right)$, the laser was used at maximum bandwidth with a spectrum shown in the inset of Fig. 1. To provide a background free detection of the CARS signal in the range of 630-700 nm, the short wavelength tail of the laser was cut by a Schott RG9 filter of $6 \mathrm{~mm}$ thickness. The laser is split into Pump and Stokes pulses by a dichroic mirror (Chroma 770DCXRU, s-polarization). To compensate the different group-velocity dispersion (GVD) of glass for the two pulses, the Stokes propagates through a thickness $L_{1}$ of SF57 glass before it is recombined with the Pump with an adjustable delay $t_{0}$ in a second dichroic mirror of equal type. Both pulses then propagate through a thickness $L_{2}=2 \mathrm{~cm}$ of SF57 and enter the microscope setup described in Ref. 12. The resulting Pump and Stokes spectra are centered around 750 and $850 \mathrm{~nm}$, respectively, as shown in the inset of Fig. 1. The total power transmission of this beam preparation can be up to $100 \%$, and is about $40 \%$ in the present setup. The additional GVD accrued by the pulses up to the sample, mainly due to the microscope objective (Leica 1.2NA water immersion), is equivalent to a length $L_{\mathrm{MO}}=2 \mathrm{~cm}$ of SF57 glass. Choosing $L_{1}=8 \mathrm{~mm}$, the linear chirp of Stokes and Pump is equal, so that a constant IFD is achieved. The effect of higher-order dispersion on the IFD matching is discussed in Ref. 14, and creates for the present case an IFD variation of $0.5-10 \mathrm{~cm}^{-1}$ over the pulse duration, depending on $t_{0}$. The Pump pulse is chirped to a duration (intensity full width at half maximum) of $\sim 1 \mathrm{ps}$, corresponding to a Fourier limit of the spectral resolution of the vibrational excitation ${ }^{14}$ of $21 \mathrm{~cm}^{-1}$, larger than, and thus not limited by, the higher order effects. The CARS created in the sample is collected by a microscope objective, transmitted through a bandpass filter (triple stack of Omega XF3081, 615-695 nm), and detected by a photomultiplier (Hamamatsu H7422-40).

To demonstrate experimentally the spectral resolution and range accessible with this excitation, we measured CARS from polystyrene (PS) beads of $3 \mu \mathrm{m}$ diameter, immersed in a gel of $2 \%$ agarose and $98 \%$ water. CARS images of a cross section through the center of a bead are shown in the insets of Fig. 2. CARS spectra indicated in Fig. 2(a) were obtained from line-scans across the bead as function of $t_{0}$, to measure both the nonresonant CARS from the gel and the CARS from PS. The nonresonant CARS shows the spectral range over which the IFD is tuneable by the delay, centered around $1600 \mathrm{~cm}^{-1}$, and covering roughly the region $1000-2000 \mathrm{~cm}^{-1}$. This range can be extended to the $2700-3100 \mathrm{~cm}^{-1} \mathrm{CH}$ region by using a spectrally broader laser source, which is commercially available. In the CARS from PS, several features are visible at spectral positions corresponding to the Raman resonances ${ }^{15}$ of PS.

In order to retrieve the CARS spectrum, corrected by the changing temporal overlap between Pump and Stokes, we evaluated the ratio between CARS on PS and the nonresonant CARS in the gel, given in Fig. 2(b). Most of the vibrational resonances show a dispersive shape in the CARS ratio due to the interference with a dominant nonresonant CARS contribution of PS. Moreover, the observed CARS ratio is increasing with decreasing $t_{0}$ (and IFD), even though the related Raman intensities (proportional to the imaginary part

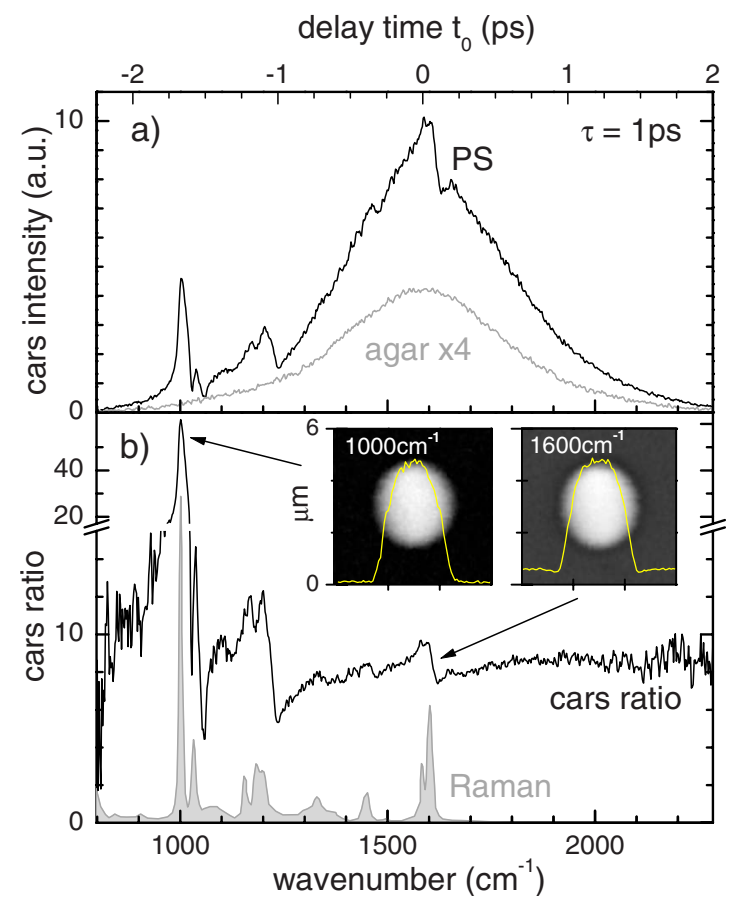

FIG. 2. (Color online) (a) Measured CARS in PS (black) and agar (gray) as function of the delay $t_{0}$. (b) CARS ratio determined from (a) and spontaneous Raman spectrum (Ref. 15) of PS. The images are lateral sections of the $3 \mu \mathrm{m}$ PS bead for IFDs as labeled. Linear gray scale from zero (black). $61 \times 61$ pixels at $0.1 \mathrm{~ms} /$ pixel. The yellow lines are horizontal cuts through the bead center. Pump power $7 \mathrm{~mW}$, Stokes power $8 \mathrm{~mW}$.

of the CARS susceptibility) are not showing such a trend. This effect is strongest for the $1001 \mathrm{~cm}^{-1}$ resonance (out-ofplane $\mathrm{CH}$ deformation), which is dominating the PS nonresonant background by a factor of 8 . Such a behavior is expected in spectral focusing CARS due to the time ordering of Pump and Stokes, which is increasing the ratio of resonant to nonresonant contributions with decreasing $t_{0}$, i.e., for a Pump pulse arriving after the Stokes pulse [see Fig. 5a in Ref. 14]. The CARS images of the PS beads [see Fig. 2(b)] taken at $1000 \mathrm{~cm}^{-1}$ thus show a better contrast than at $1600 \mathrm{~cm}^{-1}$.

We have recently developed a differential CARS (D-CARS) method, ${ }^{16}$ which is a dual-frequency extension of spectral focusing. In short, the Stokes-Pump pulse pair is split into two pairs, the second pair is delayed by half the laser repetition period, and recombined with the first pair. By adding a SF57 glass block of length $d$ to the path of the second pair, its IFD $\left(\mathrm{IFD}_{2}\right)$ is reduced compared to the one of the first pair $\left(\mathrm{IFD}_{1}\right)$ by the temporal delay introduced between Pump and Stokes due to their different group velocities. The difference of the CARS signals from the two pulse pairs is measured using the ac component of the photomultiplier current filtered by a lock-in (SRS 844) at the laser repetition rate. We also simultaneously acquire the dc component giving the sum of the CARS signals. This method eliminates the spectrally constant nonresonant CARS background and improves the CARS image contrast and chemical sensitivity. We have added to the setup in Fig. 1 the D-CARS pulse duplication unit described in Ref. 16. Transmission through polarizing beam splitters in this unit adds $20 \mathrm{~mm}$ of SF2 glass, which increases the linear chirp yielding a pulse duration of $1.3 \mathrm{ps}$, and we use $L_{1}=12 \mathrm{~mm}$ to match the linear chirp of Pump and Stokes. The resulting Fourierlimited spectral resolution is $16 \mathrm{~cm}^{-1}$. 


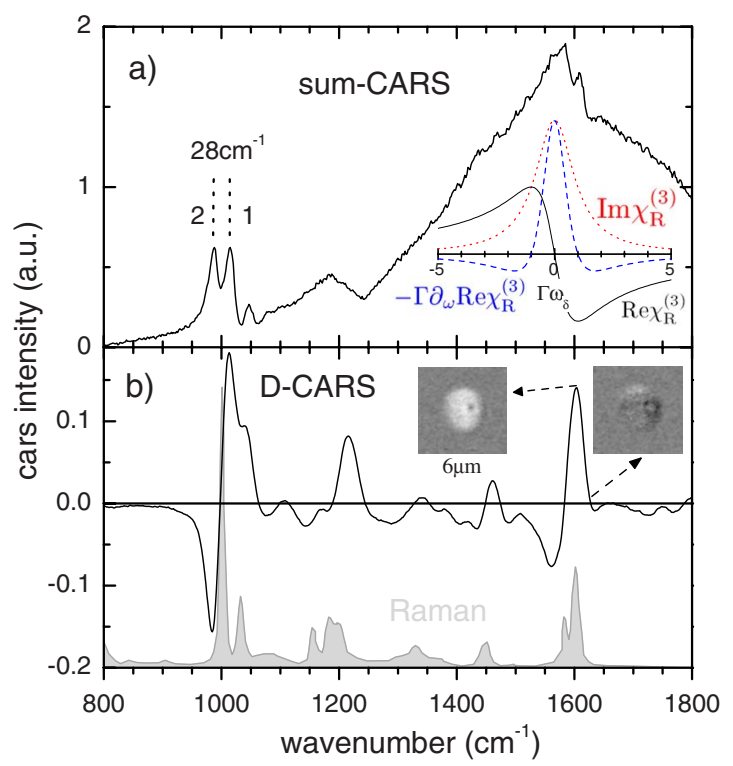

FIG. 3. (Color online) (a) Sum-CARS intensity from a PS bead versus $\left(\mathrm{IFD}_{1}-\mathrm{IFD}_{2}\right) / 2$. The difference $\mathrm{IFD}_{1}-\mathrm{IFD}_{2}$ is indicated by vertical lines. Inset: CARS resonant line-shapes (see text). (b) D-CARS corresponding to (a). Bead cross-sections are shown at $1600 \mathrm{~cm}^{-1}$ (left) and $1623 \mathrm{~cm}^{-1}$ (right) on a gray scale from -0.07 (black) to 0.14 (white). Other conditions as in Fig. 2.

When the resonant CARS contribution is smaller than the nonresonant background, which is for example the case for small concentrations in solution, the CARS lineshape is dominated by the interference $3 \chi_{\mathrm{NR}}^{(3)} \operatorname{Re} \chi_{\mathrm{R}}^{(3)}$ of the resonant third-order susceptibility $\chi_{\mathrm{R}}^{(3)}=R /\left(-\omega_{\delta}-i \Gamma\right)$ with the nonresonant one $\chi_{\mathrm{NR}}^{(3)}$. Here $\omega_{\delta}$ is the difference between vibrational excitation and resonance frequency, ${ }^{14}$ and $\Gamma$ is the vibrational dephasing rate. This creates a dispersive lineshape $\operatorname{Re} \chi_{\mathrm{R}}^{(3)}=-R \omega_{\delta} /\left(\omega_{\delta}^{2}+\Gamma^{2}\right)$ [see inset Fig. 3(a)], which is spectrally extended with tails $\propto \omega_{\delta}^{-1}$. Hence single-frequency CARS is less suited to create specific vibrational contrast than spontaneous Raman, which has a lineshape proportional to $\operatorname{Im} \chi_{\mathrm{R}}^{(3)}=R \Gamma /\left(\omega_{\delta}^{2}+\Gamma^{2}\right)$. The spectral differential $\partial_{\omega_{\delta}} \operatorname{Re} \chi_{\mathrm{R}}^{(3)}=R\left(\omega_{\delta}^{2}-\Gamma^{2}\right) /\left(\omega_{\delta}^{2}+\Gamma^{2}\right)^{2}$ however has a shape similar to spontaneous Raman, and even features half the linewidth. By measuring the difference of CARS between two close lying IFDs, we can thus recover a spectral response of high specificity, similar to stimulated Raman scattering. ${ }^{17}$ This difference is measured by D-CARS when using a small thickness $d=1 \mathrm{~mm}$, resulting in $\mathrm{IFD}_{1}-\mathrm{IFD}_{2}=28 \mathrm{~cm}^{-1}$, comparable to the spectral resolution and the relevant Raman linewidths. The corresponding sum-CARS on PS beads [see Fig. 3(a)] shows a duplication of the peaks due to the pres- ence of the two IFDs (compare with Fig. 2). The D-CARS [see Fig. 3(b)], corrected to represent relative pulse pair intensities resulting in a balanced nonresonant CARS, shows the expected peaked lineshape for the weaker Raman resonances. Cross-section images at 1600 and $23 \mathrm{~cm}^{-1}$ offresonance show the selectivity and the suppression of the nonresonant background in D-CARS. For strong resonances dominating the nonresonant background the D-CARS lineshape becomes dispersive, not suited for good specificity. In this case single CARS can be used, which is available from the measured sum and D-CARS.

In conclusion, we have demonstrated single-frequency CARS microspectroscopy using a single femtosecond laser and passive optical elements which are stable and easy to align. This method offers a spectral resolution comparable to CARS with picosecond sources, as well as fast and broadband spectral tunability, and compatibility with femtosecondlaser techniques such as SHG or TPF. With the dual frequency extension D-CARS,${ }^{16}$ the nonresonant background can be suppressed and the spectral selectivity can be equivalent to Raman or stimulated Raman scattering microscopy.

This work was funded by the U.K. Biotechnology and Biological Research Council (Grant No. BB/D001013/1).

${ }^{1}$ A. Zumbusch, G. R. Holtom, and X. S. Xie, Phys. Rev. Lett. 82, 4142 (1999).

${ }^{2}$ J.-X. Cheng and X. Xie, J. Phys. Chem. B 108, 827 (2004).

${ }^{3}$ M. Muller and A. Zumbusch, ChemPhysChem 8, 2156 (2007).

${ }^{4}$ A. Volkmer, J. Phys. D 38, R59 (2005).

${ }^{5}$ C. L. Evans and X. S. Xie, Annu. Rev. Anal. Chem. 1, 883 (2008).

${ }^{6}$ J.-X. Cheng, A. Volkmer, L. D. Book, and X. S. Xie, J. Phys. Chem. B 105, 1277 (2001).

${ }^{7}$ J.-X. Cheng, A. Volkmer, L. Book, and X. Xie, J. Phys. Chem. B 106, 8493 (2002)

${ }^{8}$ T. W. Kee and M. T. Cicerone, Opt. Lett. 29, 2701 (2004).

${ }^{9}$ B. von Vacano and M. Motzkus, Phys. Chem. Chem. Phys. 10, 681 (2008).

${ }^{10}$ A. S. Weling, B. B. Hu, N. M. Froberg, and D. H. Auston, Appl. Phys. Lett. 64, 137 (1994).

${ }^{11}$ T. Hellerer, A. M. Enejder, and A. Zumbusch, Appl. Phys. Lett. 85, 25 (2004).

${ }^{12}$ I. Rocha-Mendoza, W. Langbein, and P. Borri, Appl. Phys. Lett. 93, 201103 (2008).

${ }^{13}$ A. F. Pegoraro, A. Ridsdale, D. J. Moffatt, Y. Jia, J. P. Pezacki, and A. Stolow, Opt. Express 17, 2984 (2009).

${ }^{14}$ W. Langbein, I. Rocha-Mendoza, and P. Borri, J. Raman Spectrosc. 40, 800 (2009).

${ }^{15}$ Polystyrene Raman spectrum http://www.sigmaaldrich.com/.

${ }^{16}$ I. Rocha-Mendoza, W. Langbein, P. Watson, and P. Borri, Opt. Lett. 34, 2258 (2009).

${ }^{17}$ C. W. Freudiger, W. Min, B. G. Saar, S. Lu, G. R. Holtom, C. He, J. C. Tsai, J. X. Kang, and X. S. Xie, Science 322, 1857 (2008). 